\title{
ECONOMÍA REGIONAL Y COMERCIO DE EXPORTACIÓN EN SAN JUAN DE PASTO A MEDIADOS DEL SIGLO XIX
}

\author{
REGIONAL ECONOMY AND EXPORT TRADE AT \\ SAN JUAN DE PASTO IN THE MID CENTURY XIX \\ ECONOMIA REGIONAL E COMÉRCIO DE EXPORTAÇÃO \\ EM SAN JUAN DE PASTO EM MEADOS DO SÉCULO XIX
}

\author{
Por: Diego Andrés Quintero Timaná
}

Licenciado en Educación Básica con Énfasis en Ciencias Sociales, Universidad de Nariño; miembro del grupo de investigación Edu-Multiverso. -Universidad de Nariño- mail: diegoaqt92@gmail.com

\section{RESUMEN}

El presente escrito tiene como objetivo analizar los aspectos socio-económicos que acaecieron en San Juan de Pasto posterior a las guerras de independencia, para así examinar las características que poseía la economía local durante el auge exportador presentado en Colombia a mediados del siglo XIX. Al estudio, se incorpora la propuesta de la no simultaneidad de lo simultáneo del autor Carlos Rincón, como planteamiento que posibilita un análisis articulado entre el acontecer nacional y las particularidades regionales.

Este trabajo se orientó bajo una metodología de revisión documental sobre lo cual se efectuó un estudio de contenido a fuentes bibliográficas en torno a la historia social y económica del suroccidente colombiano, además de un estudio de fuentes archivísticas encontradas en centros de documentación histórica. Ante este proceso, se logra plantear que la ciudad de San Juan de Pasto a mediados del siglo XIX, atravesó por una debilitada situación económica generada en gran parte por las guerras de independencia y la débil articulación al auge exportador presentado en Colombia a mediados de siglo, con lo que se establece una serie de particularidades regionales ante el acontecer socio-económico nacional.

Palabras clave: Auge exportador, economía regional, guerra de independencia. JEL: R11; R51; N36. 
Diego Andrés Quintero Timaná

Economía regional y comercio de exportación en San Juan de Pasto a mediados del siglo XIX

\section{ABSTRACT}

This paper analyzes the socio-economic aspects that occurred in San Juan de Pasto after the wars of independence. Also, it examines the characteristics that had the local economy during the export apogee presented in Colombia in the mid-nineteenth century. In this study, the proposal of non-simultaneity of the simultaneous of the author Carlos Rincón is introduced which allows an analysis that connects the national events and regional particularities.

This work was oriented under a methodology of document review; making a study of content to bibliographical sources about the social and economic history in southwestern Colombia, and a study of archival of sources found in historical documentation centers. After, of this process is found that the city of San Juan de Pasto in the mid-nineteenth century went through a difficult economic situation generated by the wars of independence and the fragile link in the export apogee presented in Colombia in mid-century. Therefore, regional particularities before the national socio-economic events were established.

Keywords: Apogee exporter, regional economy, war of independence.

JEL: R11; R51; N36.

\section{RESUMO}

Este artigo analisa os aspectos socioeconômicos que ocorreram em San Juan de Pasto após as guerras de independência para examinar as características que tinham a economia local durante o apogeu de exportação apresentados na Colômbia em meados do século XIX. Neste estudo, a proposta de não simultaneidade da simultânea do autor Carlos Rincón é introduzida, permitindo uma análise ligada entre eventos nacionais e particularidades regionais.

Este trabalho foi orientado sob uma metodologia de revisão de documentos; fazendo um estudo de conteúdo a fontes bibliográficas sobre a história social e econômica no sudoeste da Colômbia, e um estudo de arquivamento de fontes encontradas em centros de documentação histórica. Após este processo se constatar que a cidade de San Juan de Pasto, em meados do século XIX passou por uma difícil situação econômica gerada pelas guerras de independência e o elo frágil no apogeu de exportação apresentados na Colômbia em meados do século; Por conseguinte, foram estabelecidas particularidades regionais antes dos eventos socioeconômicas nacionais.

Palavras-chave: Apogeu de exportação, da guerra de independência, economia regional.

JEL: R11; R51; N36. 


\section{INTRODUCCIÓN}

Las primeras décadas del siglo XIX en Colombia estuvieron caracterizadas, entre otros aspectos, por el proceso de independencia ante España, proyecto que significó una considerable pérdida de vidas además de la inestabilidad económica, política y social para la naciente república. En materia económica, los elevados gastos en materiales de guerra, el continuo robo de productos, cosechas y ganado para el mantenimiento de las tropas, y el detrimento de la agricultura y el comercio debido a la falta de recursos económicos y la escasez de mano de obra, hicieron que el transcurso de las guerras de independencia dejaran una serie de secuelas en la dinámica económica del país durante la primera mitad del siglo XIX.

Ante el desequilibrio de la economía nacional que había dejado el proceso de independencia, los gobiernos liberales de medio siglo establecieron políticas económicas como el Librecambio, que tenían como objetivo transformar la herencia del sistema económico colonial e introducir al país a las dinámicas comerciales de los mercados internacionales predominantes en la época; en este contexto, se presenta en Colombia un auge agroexportador de productos como tabaco, algodón, quina, añil, caucho, tagua, cacao y café, los cuales generaron ingresos económicos favorables a las regiones productoras.

En este sentido, si bien existe una considerable colección bibliográfica en torno a la historia económica de la primera mitad del siglo XIX en Colombia, la cual se ha enfocado en examinar el proceso de transición de la inestabilidad económica, acaecida posterior a las guerras de independencia, al crecimiento económico que significó para el país el auge agroexportador de mediados de siglo, es conveniente indagar acerca de las diversas particularidades presentadas en los espacios regionales. Es por ello, que el presente escrito pretende analizar aspectos socio-económicos de San Juan de Pasto posterior al proceso de independencia, así como también la inserción de la economía local en el auge exportador presentado en Colombia a mediados del siglo XIX, incorporando al análisis la propuesta de la no simultaneidad de lo simultáneo del autor Carlos Rincón, como planteamiento que posibilita un análisis entre el acontecer histórico nacional y las particularidades regionales.

La propuesta de la no simultaneidad de lo simultáneo, proporciona un esquema para la comprensión de procesos y fenómenos particulares en regiones y subregiones, que confrontan los estereotipos de una imagen nacional simultánea y homogénea. En este proceso, bajo la presión ejercida para la construcción de identidades nacionales por parte del proceso de globalización, los estudios sobre heterogeneidad y diversidad social y cultural se hacen prioritarios en el reconocimiento de las particularidades anuladas e invisibilizadas, es decir lo no simultáneo (Rincón, 1995: 218).

Si bien, con el proyecto de la modernidad se fortalece la configuración de la simultaneidad y de la sincronía nacional o global como uno de los mecanismos para cohesionar a la población bajo un mismo marco de referencia histórica, política y económica, el planteamiento de la no simultaneidad de lo simultáneo, se enfoca en examinar los ritmos diferentes, los casos particulares, la existencia de otros espacios y sincronías ante los expuestos como referentes de simultaneidad u homogeneidad discursiva. 
Por ello en el artículo se desarrolla un ejercicio de reelaboración discursiva que articule los acontecimientos regionales acaecidos en relación a la temática de estudio como parte de las múltiples dinámicas que conforman el entramado histórico nacional, el cual ha sido predominado por relatos homogéneos centralizados, negando la participación de las características específicas de las regiones y subregiones del país.

Por otra parte, este trabajo se realizó a partir de un enfoque histórico-hermenéutico, orientado bajo un método de revisión documental sobre lo cual se efectuó un estudio de contenido a fuentes bibliográficas en torno a la historia económica del suroccidente colombiano, así mismo, se realizó una búsqueda y posterior estudio de fuentes hemerográficas y archivísticas en centros de documentación histórica como el Instituto Municipal Archivo Histórico de Pasto, en donde se analizó fuentes primarias como archivos notariales, decretos, libros de cabildo, documentación de tipo periodístico, entre otros documentos en relación a la temática de estudio.

Por último, el artículo plantea en un primer momento identificar las características socio-culturales de San Juan de Pasto a mediados del siglo XIX, posteriormente examinar la incidencia de los conflictos civiles acaecidos por el proceso de independencia en la dinámica económica regional, y finalmente examinar la participación de la economía de San Juan de Pasto en el comercio de exportación durante el periodo de estudio; siendo estas partes las que conforman la estructura del documento.

\section{LA POBLACIÓN DE SAN JUAN DE PASTO A MEDIADOS DEL SIGLO XIX}

Entre las características sociales de las ciudades colombianas durante la mitad del siglo XIX, se encuentra un estilo de vida que se veía alterado por los días de mercado, las festividades religiosas y las confrontaciones civiles que interrumpían notablemente la rutina cotidiana de los centros urbanos, entre otros aspectos que han sido planteados por la historiadora Beatriz Castro Carvajal (1996: 241).

Así pues, San Juan de Pasto comparte estas similitudes en las dinámicas sociales que se presentaron en gran parte de las ciudades republicanas. La ciudad en mención, a mediados del siglo XIX, se caracterizó por albergar una población que conserva una tendencia a mantener lo tradicional y la poca aceptación de lo nuevo, además de su actitud desconfiada y recelosa frente a lo desconocido, según lo manifiesta el autor Chaves Chamorro (1983: 201).

En relación a las características culturales e ideológicas de la sociedad pastusa durante el siglo XIX, Benhur Cerón Solarte y Marco Tulio Ramos expresan que dichas características se encuentran enmarcadas dentro del parámetro colonial, dominado por una atmósfera religiosa que penetra todas las esferas sociales y las manifestaciones espirituales de sus habitantes (1997: 198). En concordancia con este planteamiento, historiadores como Karol Viviana Luna Zarama y Gerardo Guerrero manifiestan:

En Pasto los elementos de cohesión social que colaboraron en el proceso de construcción de su identidad cultural, que modelaron las costumbres, el tipo de relaciones sociales y económicas y el paradigma predominante entre los 
grupos sociales de esta provincia fueron: 1. La iglesia Católica y 2. La Educación. Es en esta región, donde la iglesia católica encontró el espacio adecuado para sembrar sus principios y hacerlos florecer en su máximo esplendor. Para los dirigentes conservadores de la sociedad pastusa, la Iglesia Católica era el dispositivo más importante para mantener el orden social y moral de las comunidades, es ella quien guiaba las pautas de comportamiento ético y moral de los ciudadanos (2011: 27).

Estos referentes sociales permiten establecer que las manifestaciones culturales gestadas desde la época colonial, continúan vigentes en gran parte de la población de Pasto a mediados del siglo XIX, viéndose reflejadas dichas manifestaciones en las fiestas religiosas heredadas de la colonia, las cuales, como lo indican los autores Cerón y Ramos, son espacios de tregua dentro del intrincado laberinto político, que da paso también al fomento de negocios de fermentación y destilería; siendo las fiestas en la que cada parroquia o convento busca protagonismo distribuyéndose las celebraciones a lo largo del año entre los respectivos santos patrones (1997: 208).

El autor Benhur Cerón Solarte manifiesta que el poder civil y eclesiástico en la ciudad de Pasto, representa una sociedad profundamente religiosa, cuyas expresiones del mundo material en el espacio geográfico se materializan a través de grandes catedrales y conventos que dominan el paisaje:

Tal es el papel de las iglesias que culturalmente se conciben como "lugar central" a partir del cual se organiza el espacio circundante; en este ordenamiento territorial, la distancia hacia el lugar central, es decir la plaza de la Constitución, constituye la variable fundamental que explica la lógica de la ubicación en el paisaje asociada al ejercicio del poder en un contexto determinado (1996: 57).

Otro de los indicadores que permite caracterizar la dinámica social de la ciudad de Pasto como un espacio en el que se mantiene un orden social y moral, se hace visible en la circular No. 7 del mes de febrero de 1860, emanada por la gobernación de la provincia, en la cual se indica al alcalde municipal del distrito de Pasto algunas recomendaciones acerca de mantener el orden público, la moralidad y tranquilidad de la población, según se muestra a continuación:

El primer deber de un empleado celoso de la buena marcha de los pueblos, cuya administración se lo ha encargado, es el procurar el buen arreglo y el comportamiento moral de todos los ciudadanos; para que de ellos dependa el orden, la obediencia de las leyes, la seguridad de las personas y de las propiedades. Por estas razones recomiendo a usted que haciendo publicar en la alcaldía de su mando la ley general y ordenanza de policía y la ley sobre vagos, mande usted a encarcelar a todos los individuos que careciendo de medios de subsistencia no se dedican a ninguna clase de oficio ni ocupación honesta que les produzca lo necesario para vivir; que haga enjuiciar a todos los que escandalosamente vivan y los que andan por las calles diciendo palabras obscenas y alborotando. 
Diego Andrés Quintero Timaná

Economía regional y comercio de exportación en San Juan de Pasto a mediados del siglo XIX

En esto debe poner usted el mayor empeño y cuidado porque de ello depende precisamente el que todos los ciudadanos se consagren al trabajo, se ocupen de medios decorosos para mantenerse, y también que respeten los bienes de sus semejantes, que es una cosa esencialmente social y de eminente necesidad para que haya tranquilidad y progreso en los pueblos. Usted sabe que por desgracia, la desmoralización que corrompe las costumbres y el libertinaje, son los males más funestos, el azote más terrible que podría sobrevenirnos, pareciéndose a la guerra, la peste y el hambre, destruyendo la esperanza de tener tranquilidad en los pueblos, en las casas y en las familias (Instituto Municipal Archivo Histórico de Pasto, 1860: Caja No. 40, Libro: 01, Folio: 223 y 224).

Las pautas de comportamiento social establecidas en el anterior enunciado, son muestra del interés que tenía la iglesia católica y la élite política en su mayoría conservadora, por vigilar y modelar ética y moralmente a la sociedad civil en Pasto; por ello, la alcaldía y la policía del distrito, eran las instituciones encargadas de vigilar que se cumpla el buen comportamiento civil, mientras que la iglesia se encargaba de inspeccionar el orden moral de los habitantes.

Estas características sociales de la población de San Juan de Pasto, se encuentran inmersas en una región sumamente tradicional como lo fue el Estado Soberano del Cauca, el cual estaba compuesto por antiguas familias terratenientes que se negaban a perder la importancia política y social que habían tenido hasta los inicios de la república. La consolidación de un poder regional se había dado desde la colonia, época en la cual las clases dominantes tenían un estrecho marco localista, lo que les permitía controlar los órganos locales de poder; al respecto el autor Alonso Valencia Llano señala:

Durante el siglo XIX, el desarrollo económico, político y social colombiano se dio en medio de una serie de conflictos que obedecían a intereses regionales, los cuales hacen difícil hablar de la "nación colombiana", siendo más sencillo referirse a los Estados Federales o Soberanos, lo que da una idea del alcance de la cuestión regional. [...] Se estaría así ante la existencia de una fragmentación de las clases dominantes, que se prolongaría desde la colonia hasta el siglo XIX; proceso en el cual se presentaron intentos de unificación nacional que produjeron choques políticos que terminaron en guerras civiles de carácter supraregional. De hecho, los choques políticos suscitados por la necesidad de solucionar la "cuestión regional" muestran la fortaleza de las elites provincianas y la debilidad del gobierno central (1988: 14).

Las diferencias políticas que se presentaron entre los diferentes Estados que conformaban el país, al igual que las confrontaciones civiles ocurridas al interior de los Estados, iban a generar un ambiente regional marcado por conflictos ideológicos y militares. En este sentido, durante el periodo del Liberalismo Radical en Colombia, se presentaron una serie de enfrentamientos, los cuales se examinarán detalladamente más adelante, cuyas consecuencias repercutirían en la inestabilidad política y económica de San Juan de Pasto, que hace parte también del entramado social que caracterizó a la sociedad pastusa durante el periodo de estudio. 
Las confrontaciones bélicas acaecidas a lo largo del siglo XIX, las cuales hicieron parte de la vida cotidiana de los pobladores de Pasto; aproximadamente 9 guerras nacionales, 14 regionales y un sinnúmero de locales, según lo plantea el historiador Pedro Verdugo (2001: 1), tienen sus orígenes en las contiendas electorales, la polarización política entre liberales y conservadores, el poder hermético de la iglesia católica, los modelos de organización y manejo del Estado, la presencia de poderes regionales que impedían la consolidación de una cohesión social nacional, y la existencia de un país regionalizado y con escasa conexión entre sus territorios, son entre otros aspectos, algunos de los factores que influyeron en las numerosas confrontaciones que demarcaron el acontecer social nacional y regional durante el siglo XIX.

Es menester tener en cuenta, que la participación de Pasto en estas confrontaciones civiles, estuvo relacionada con el aporte de dinero proveniente de impuestos de guerra, así como también con la incorporación de hombres a las fuerzas militares y los considerables aportes en bienes para albergar y alimentar a las tropas en las épocas de conflicto. Ante estas dinámicas sociales, la población de San Juan de Pasto se encontraba en un contexto marcado por el dominio cultural de la iglesia católica y por las repercusiones sociales y económicas de las confrontaciones civiles que hicieron parte del acontecer nacional y regional.

\section{CONFLICTOS CIVILES Y ECONOMÍA REGIONAL EN LA PRIMERA MITAD DEL SIGLO XIX}

Uno de los conflictos que marcó la historia nacional a inicios del siglo XIX fue el proceso de independencia ante España. Anterior a este proceso, el virreinato de la Nueva Granada había obtenido un crecimiento económico notable durante la segunda mitad del siglo XVIII, el cual se frenó a partir de 1808 con el colapso de España, invadida por Napoleón. El crecimiento económico del país se tornó negativo posteriormente por la interrupción del comercio debido a las guerras de independencia, el deterioro del esclavismo y el estancamiento del comercio internacional que se presentaría hasta 1850, según lo indica el autor Salomón Kalmanovitz (2008: 10).

Sin embargo, durante la primera mitad del siglo XIX, el proceso de independencia dejó una serie de consecuencias que configuran una simultaneidad a nivel nacional como lo fue la inestabilidad política, los frecuentes conflictos civiles y el estancamiento económico presentado en diversas regiones del entramado nacional que duró hasta mediados del siglo. Además, entre los costos de las guerras de independencia cabe mencionar la destrucción de vidas, de viviendas, de bienes productivos como reses, mulas y caballos, además de los elevados gastos económicos en materiales de guerra.

$\mathrm{Al}$ respecto, Kalmanovitz plantea:

En efecto, lo más costoso a largo plazo del proceso independentista sería la inestabilidad política. El cambio frecuente de las reglas constitucionales afectaba los planes de los empresarios que surgieron de la matriz colonial, y sus negocios difícilmente prosperaron. Comercios, minas y haciendas queda- 
ban sometidos a riesgos de expropiación, de préstamos forzados durante los conflictos y de cambios frecuentes en las reglas de juego que organizaban la sociedad. Colombia tuvo nueve constituciones durante el siglo XIX y fue uno de los países más inestables de la órbita hispanoamericana, lo cual incidiría en el trascurrir económico nacional (2010: 73).

Así mismo, autores como William Paul McGreevey, señalan que posterior a la etapa de independencia, varias de las características económicas del sistema colonial permanecieron vigentes; al no surgir otros productos de exportación, continuaba la dependencia del país en la explotación de las minas de oro para obtener divisas extranjeras, los sistemas de transporte y de comunicación nacional permanecían en mal estado, así como también se presentaba un fraccionamiento interno en el mercado de alimentos (1989: 8).

Esta serie de características, retomando los planteamientos del autor Carlos Rincón, configuran un entramado de simultaneidad de lo que representó las guerras de independencia para la dinámica económica del país. En este sentido, acorde a los planteamientos metodológicos del autor, se trae en mención procesos sociales particulares que permitan establecer los modos de articulación entre la región o subregión de estudio con los procesos de continuidad discursiva o simultaneidad enmarcados en una mayor escala.

Por lo anterior, la región del suroccidente colombiano se acopla a los procesos de simultaneidad acaecidos a nivel nacional en relación a las consecuencias económicas de las guerras de independencia. La economía regional había sido afectada notoriamente, puesto que el trabajo sobre la agricultura y la ganadería se paralizó debido a la falta de recursos económicos, la escasez de mano de obra y el continuo robo de productos, cosechas y ganado para el mantenimiento de las tropas inmersas en el conflicto.

En el caso particular de la ciudad de San Juan de Pasto, los destrozos de las guerras tuvieron como consecuencia la muerte o ausencia de hombres en edad productiva, los cuales, unos fueron reclutados a la fuerza y otros huyeron voluntariamente, siendo los hombres el principal sostén económico de muchas familias que quedaron en una debilitada situación económica. Así mismo, las tropas que entraron a la ciudad, bien sea a "nombre del rey" o a "nombre de la república", cometieron todo tipo de abusos y saqueos en la ciudad y en campos aledaños tal como lo indica el historiador Gerardo Guerrero; además, se debe tener en cuenta las confiscaciones de las cajas reales que dejaron sin fondos al erario público y las contribuciones obligatorias de la población que paulatinamente fue sometida a una mayor desolación y pobreza (2000: 210).

$\mathrm{Al}$ respecto, el historiador Sergio Elías Ortiz manifiesta:

Las medidas más drásticas en materia económica contra el pueblo pastuso las aplicó Bolívar y su lugarteniente Bartolomé Salom. Mediante el decreto del 13 de enero de 1823 ordenó la confiscación de bienes de un gran número de habitantes de Pasto que habían apoyado el levantamiento protagonizado por Agustín Agualongo; por lo cual, creo una comisión para la repartición de las propiedades confiscadas y varias de las mejores haciendas pasaron a manos de los militares: Panamal, El Salado, Aranda, Santa Mónica, San Guillermo 
y Simarrones, fueron expropiadas por el hecho de que sus propietarios se declararon fieles al juramento de fidelidad al rey. Además, se impuso la elevada suma de 30.000 pesos como sanción a la ciudad, obligó a reunir 300 caballos y algo más de mil reses para llevarlas a Quito y exigió contribuciones individuales a los personajes más acaudalados de la ciudad (1958: 458).

Este periodo de conflictos generó una desfavorable balanza económica para diversas regiones del país, especialmente, la provincia de Pasto que se había inclinado por la causa realista; ésta fue una de las regiones que terminó particularmente destruida, con su población diezmada y con notables secuelas económicas en la población como la baja capacidad de compra que se produjo a raíz de la escasez de moneda; lo que permite plantear que el proceso de independencia, al igual que en otras regiones del país, representó para la ciudad de Pasto inestabilidad política y desequilibrio económico; factores que incidirían en la débil articulación de la economía local al auge exportador que se produjo en Colombia a mediados del siglo XIX.

\section{SAN JUAN DE PASTO EN EL COMERCIO DE EXPORTACIÓN A MEDIADOS DEL SIGLO XIX}

Si bien el proceso de independencia significó un desequilibrio en la economía nacional, a mediados del siglo XIX se dio paso a la implementación de libertades comerciales que buscaban transformar el legado del sistema económico colonial, mediante la vinculación del país con el mercado mundial durante el periodo que se extiende entre 1850 a 1886. En este sentido, al examinar las tasas de crecimiento económico de Colombia para la primera y segunda mitad del siglo XIX, es posible considerar el impacto del proceso de independencia sobre el estancamiento económico de la primera mitad del siglo y la posterior recuperación a partir del auge exportador de los años cincuenta, como se muestra en la siguiente gráfica:

Gráfico 1. PIB por habitante, Colombia: 1765 - 1905

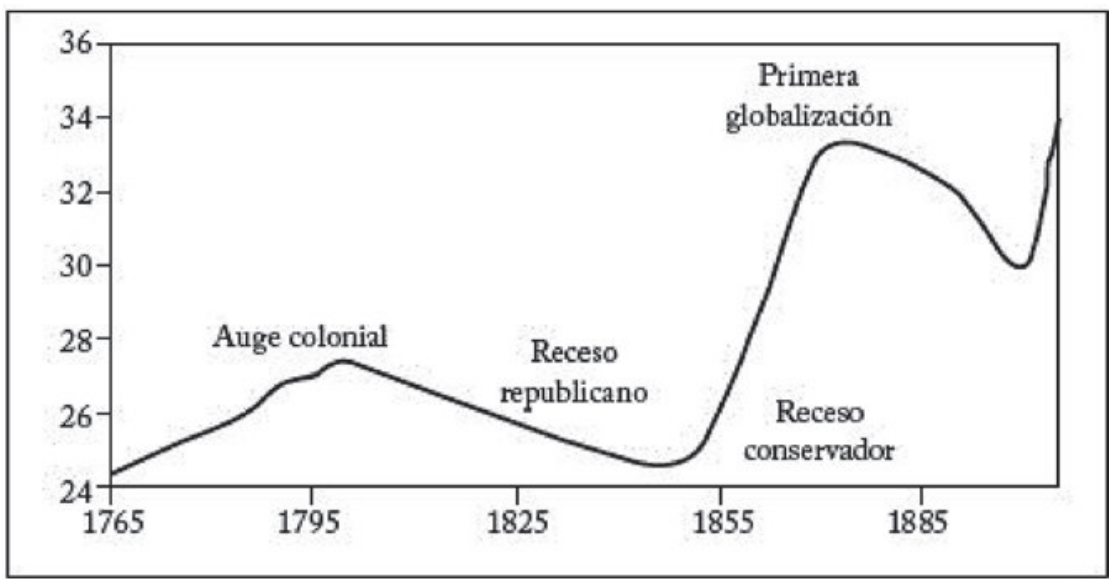

Fuente: Kalmanovitz, 2010: 76. 
Los índices del PIB presentados en la gráfica anterior, dejan entrever tres etapas a tener en cuenta: la primera es el auge colonial presentado a finales del siglo XVIII, lo que refleja que la última fase colonial fue de relativa prosperidad económica para gran parte del Virreinato de la Nueva Granada; posteriormente la segunda etapa denominada como receso republicano, estuvo marcada por el debilitamiento económico que produjo las guerras de independencia y la inestabilidad política del país, en un contexto en el cual el comercio, las minas y haciendas quedaban sometidas a riesgos de expropiación o de préstamos forzados para solventar los gastos de las guerras; y por último, aparece la etapa denominada como primera globalización, en la cual las reformas liberales de medio siglo pretendieron reanudar la dinámica comercial del país sobre una base exportadora de materias primas que posibilitó un crecimiento en las índices económicos nacionales.

El historiador británico Eric Hobsbawn, plantea que la etapa que va desde 1848 hasta 1870, se caracterizó por la rápida transformación y expansión económica, como reflejo de la consolidación del sistema capitalista en el mundo. En la segunda mitad del siglo XIX, la industrialización creció de forma vertiginosa, se ampliaron las relaciones comerciales entre los países generando que, entre otros aspectos, el comercio mundial entre 1850 a 1870 aumentara en un 260\% en comparación con las dinámicas comerciales de inicios del siglo XIX (1998: 43).

Los procesos de apertura económica gestados a mediados del siglo XIX, significó para los países industrializados de Europa, el camino para vender libremente sus mercancías a bajos precios en todos los mercados del mundo, mientras que, los países con un menor avance industrial, como los de América Latina, vendieron materias primas, principalmente agrícolas, en grandes cantidades y a bajos precios como la alternativa económica para ingresar al sistema económico capitalista imperante en la época.

Por otra parte, para el autor José Antonio Ocampo, entre los factores que motivaron a los gobiernos liberales del siglo XIX a implementar la política económica Librecambista, se encuentran las características coloniales de la economía colombiana. Estas características estaban configuradas por una débil articulación al mercado mundial y el escaso desarrollo del mercado interno, con lo cual no significa que no existieran intercambios a largas distancias, ni tampoco que en todo el país predominara la producción para el autoconsumo, sino más bien que las redes mercantiles existentes estaban restringidas por las características geográficas del territorio nacional y el precario sistema de vías de comunicación (2013: 12).

La apertura económica implementada a mediados del siglo XIX tuvo un efecto notable para la economía del país, a pesar de las dificultades en materia de vías de comunicación que se presentaba en gran parte de las regiones, se logró aprovechar el auge del mercado internacional y se exportó diversos productos. Estas características componen la simultaneidad económica nacional, contexto en el cual se fomentaron cultivos de exportación como tabaco, algodón, cacao y café, generando ingresos económicos favorables a las regiones productoras. 
Si dirigimos el análisis hacia la economía del Estado del Cauca al cual pertenecía la ciudad de San Juan de Pasto, autores como Alonso Valencia Llano ponen en evidencia que tras la implementación de las políticas liberales de medio siglo, se presentó un ciclo de bonanza económica en el cual prosperaron renglones económicos dedicados a la producción de tabaco, cacao, azúcar, quina, tagua y caucho que en esos momentos tenían una destacada demanda en el comercio internacional, dejando a su vez, ganancias para los comerciantes y campesinos dedicados a su producción (1998: 121).

Son varias las subregiones del Estado del Cauca que se integran a la simultaneidad nacional en el propósito de fomentar la búsqueda de nuevos cultivos con posibilidades de exportación y lograr así recuperar la estabilidad económica que los conflictos civiles habían desestabilizado. Sin embargo, la participación de la ciudad de Pasto en este proceso agroexportador es limitada, puesto que gran parte de los cultivos de clima frío se producen en Europa, lo que hizo que la demanda internacional de productos cultivados en esta región tengan una disminuida demanda en el comercio internacional, además, las regiones que poseen productos de extracción como el piedemonte amazónico, eran de difícil acceso debido a la carencia de vías, lo que representaba altos costos en el transporte de los productos.

Autores como Benhur Cerón y Marco Tulio Ramos, plantean que como consecuencia del proceso de inserción a la economía internacional, aparecen nuevas rivalidades entre ciudades que reacomodan los lazos de influencia con otros centros de poder económico, o luchan por mantener anexas áreas mineras o proveedoras de productos exportables. En estas circunstancias, la ciudad de Pasto afronta drásticas modificaciones en su antiguo estatus, puesto que pasó de ser un centro de notable importancia regional, en aspectos sociales, políticos y económicos en la época colonial, a convertirse en una ciudad de limitada trascendencia en la época republicana, afectada por la guerra, menospreciada por su defensa al proyecto realista, aislada geográficamente de los centros de poder y con una reducida participación en el auge agroexportador que tuvo el país en el siglo XIX (1997: 167).

En este sentido, la región sur del Estado del Cauca que correspondía a los altiplanos de Pasto, Túquerres e Ipiales, se enfocaron principalmente en la agricultura, tanto para el consumo interno como para el intercambio con el Ecuador y el envío a las poblaciones costeras de Barbacoas y Tumaco, además, se mantenía un contrabando significativo con el Ecuador a través de las aduanas de Carlosama y Tumaco (Valencia, 1994: 24). El comercio en la ciudad de Pasto tuvo dinámicas favorables desde la época colonial, debido a que fue paso obligado para los mercaderes y viajeros que transitaban entre Quito y Popayán, según lo manifiesta la autora Rosa Isabel Zarama:

Viajeros y comerciantes de todas las condiciones desde arrieros hasta importantes militares, aprovecharon las pequeñas comodidades que ofrecía la población para descansar de las arduas jornadas, mandar a lavar la ropa, socializar con clientes y amigos, conocidos o la elite local; mejorar su alimentación por algunos días; además de adquirir las provisiones para continuar 
el viaje como aguardiente, bizcochos, dulces, quesos y tasajo. Entre tanto las mulas y los caballos pastaban y se recuperaban de los intensos recorridos. Igualmente, la permanente presencia de militares demandaba el comercio de comida, chicha, tabaco, velas, entre otros productos (2012: 297).

Asimismo en materia de comercio agrícola, las zonas aledañas a la ciudad se dedicaban principalmente al cultivo de papas, arveja, cebolla, habas, ocas, coles, ollocos, trigo; productos que intercambiaban con poblaciones de zonas de clima cálido y templado como Sandoná, Consacá, Bomboná y Ancuya, entre otras localidades. De estos lugares, llegaban a la ciudad productos como aguacates, limones, naranjas, plátanos, piñas, además de los derivados de la caña de azúcar como melcochas, guarapo y panela, lo que posibilitaba un comercio de complementariedad entre los cultivos de zonas de clima frío y templado. Complementa la oferta comercial de la ciudad de Pasto el comercio agrícola que se sostiene por demanda de Popayán y la actual región del Valle del Cauca que acaparan la producción de harina y el ganado, especialmente las mulas de carga, también hacen parte de la oferta mercantil que sale de la región de Pasto, por la alta demanda que exige el transporte en recua entre Popayán y Quito (Cerón; Ramos, 1997: 169).

Además de la agricultura, otro de los renglones económicos de San Juan de Pasto estaba basado en la producción artesanal; Cerón Solarte y Ramos plantean que "su rentabilidad y competitividad se mantenían por disponer de pequeñas industrias desarrolladas en domicilios familiares, por la utilización de materias primas de la localidad, los bajos costos de producción y la fuerza de trabajo familiar y alta calidad” (1997: 169). Artesanos dedicados a las labores de albañilería, carpintería, herrería, platería, sastrería, zapatería, y pintura al óleo y de barniz, entre otros, junto a comerciantes y agricultores, cumplían un papel fundamental en el propósito de dinamizar la economía de la ciudad, la cual había decaído durante la época de las guerras de independencia y años posteriores.

Así pues, la débil inserción de la economía local ante el auge agroexportador, representa una de las no simultaneidades en las dinámicas económicas del país a mediados del siglo XIX. Sin embargo, cabe resaltar que a pesar de la endeble situación económica de la ciudad, diversos grupos sociales como campesinos, comerciantes, hacendados, y artesanos buscaron alternativas de fomentar la economía regional y la participación comercial en otras latitudes; con lo que se pone en cuestión la idea generalizada del aislamiento económico de Pasto en relación a las dinámicas sociales nacionales e internacionales.

\section{CONCLUSIONES}

La economía colombiana en la primera mitad del siglo XIX, estuvo caracterizada, entre otros aspectos, por el debilitamiento económico que representó las guerras de independencia ante España, y la inserción del país en la dinámica económica internacional como exportador de materias primas, generando ingresos económicos favorables a las regiones productoras. En este sentido, la ciudad de San Juan de 
Pasto se caracterizaba por albergar una mayoritaria población conservadora y católica, la cual, en el proceso de independencia había defendido el proyecto realista a diferencia de gran parte de las ciudades granadinas, causando una inestabilidad social, política y económica que marcaría su devenir histórico.

A mediados del siglo XIX, los gobiernos liberales implementaron una serie de reformas que incluía la implementación de libertades comerciales, con el fin de transformar el legado del sistema económico colonial e insertar la economía nacional a los mercados imperantes en la época. Está dinámica comercial representó para Colombia un auge agroexportador de materias primas como tabaco, quina, anís, algodón, cacao, café, entre otros, hacia países de Europa occidental y Norteamérica; sin embargo, la economía de San Juan de Pasto contó con una reducida participación en este auge agroexportador nacional, debido a factores como la escasa producción de frutos de clima tropical, la precariedad de las rutas de transporte y la relativa distancia a los puertos marítimos del norte del país.

Ante estas características, grupos sociales como campesinos, comerciantes, hacendados, y artesanos buscaron alternativas de fomentar la economía regional mediante el fortalecimiento de las manufacturas locales y una participación comercial más activa con la República del Ecuador, generando una dinámica socioeconómica particular a los procesos de simultaneidad acaecidos a nivel nacional. Es por ello, que mediante el presente escrito se pretende aportar al reconocimiento de las particularidades económicas regionales, desde las cuales se generan modos de pensar, sentir y actuar que han sido invisibilizados por los discursos de simultaneidad nacional y mundial, y que contribuyen a los procesos de renovación discursiva que está aconteciendo en las sociedades contemporáneas. 


\section{REFERENCIAS BIBLIOGRÁFICAS}

1. CASTRO, Beatriz (1996). Historia de la vida cotidiana en Colombia. Editorial Norma. Bogotá.

2. CERON, Benhur (1996). Manual Historia de Pasto. (Tomo I). Academia Nariñense de Historia. San Juan de Pasto.

3. CERÓN, Benhur y RAMOS, Marco (1997). Pasto: Espacio, Economía y Cultura. Fondo Mixto de Cultura. San Juan de Pasto.

4. CHAVES, Milciades (1983). Desarrollo de Nariño y su Universidad. Ediciones Tercer Mundo. Bogotá.

5. GUERRERO, Gerardo (2000). Manual Historia de Pasto (Tomo I). Academia Nariñense de Historia. San Juan de Pasto.

6. HOBSBAWN, Eric (1998). La era del capital: 1848-1875. Editorial Planeta, Buenos Aires.

7. INSTITUTO MUNICIPAL ARCHIVO HISTÓRICO DE PASTO. Fondo Cabildo, Año: 1860. Cajas No. 40, 41 y 42. San Juan de Pasto.

8. KALMANOVITZ, Salomón (2008). Consecuencias económicas del proceso de Independencia en Colombia. Editorial Universidad Jorge Tadeo Lozano. Bogotá.

9. KALMANOVITZ, Salomón (2010). Nueva Historia Económica de Colombia. Editorial Taurus. Bogotá.

10. LUNA, Karol y GUERRERO, Gerardo (2011). El papel político de la iglesia Católica en la provincia de Pasto: 1863-1880. Editorial Universidad de Nariño. San Juan de Pasto.

11. MCGREEVEY, William (1989). Historia económica de Colombia: 1845-1930. Tercer Mundo Editores. Bogotá.

12. OCAMPO, José (2013). Colombia y la Economía Mundial: 1830-1910. Editorial Universidad de los Andes. Bogotá.

13. ORTíZ, Sergio (1958). Agustín Agualongo y su tiempo. Editorial ABC. Bogotá.

14. RINCÓN, Carlos (1995). La no simultaneidad de lo simultáneo: Postmodernidad, globalización y culturas en América Latina. Editorial Universidad Nacional de Colombia. Bogotá.

15. VALENCIA, Alonso (1988). Estado Soberano del Cauca, Federalismo y Regeneración. Banco de la República. Bogotá.

16. VERDUGO, Pedro (2001). La guerra civil de 1876 - 1877 yel Ocaso del Liberalismo Radical en los Estados Unidos de Colombia. Crisis, Intolerancia y Clientelismo. Impresos La Castellana. San Juan de Pasto.

17. ZARAMA, Rosa (2012). Pasto: cotidianidad en tiempos convulsionados, 1824-1842. Punto Editor. San Juan de Pasto. 